\title{
Autonomous Landing System: Safe Landing Zone Identification
}

\author{
Aljaž Blažič ${ }^{1}$, Klemen Kotnik ${ }^{1}$, Kristina Nikolovska ${ }^{1}$, Miha Ožbot ${ }^{1}$, Martin Pernuš ${ }^{1}$, \\ Uroš Petkovič ${ }^{1}$, Nika Hrušovar ${ }^{2}$, Matic Verbič̌, Irena Ograjenšek ${ }^{2}$, Andrej Zdešar ${ }^{1}$, \\ Matevž Bošnak ${ }^{1}$, Tine Tomažič ${ }^{3}$, Gregor Klančar ${ }^{1 *}$ \\ ${ }^{1}$ Faculty of Electrical Engineering, University of Ljubljana, Trzaška 25, 1000 Ljubljana, Slovenia; \\ *gregor.klancar@fe.uni-lj.si \\ 2 Faculty of Economics, University of Ljubljana, Kardeljeva ploščad 17, 1000 Ljubljana Slovenia \\ 3 PIPISTREL VERTICAL SOLUTIONS d.o.o, Vipavska cesta 2, 5270 Ajdovščina, Slovenia
}

SNE 28(4), 2018, 165 - 170, DOI: 10.11128/sne.28.tn.10444

Received: November 12, 2018; Revised November 21, 2018;

Accepted: December 3, 2018

SNE - Simulation Notes Europe, ARGESIM Publisher Vienna

ISSN Print 2305-9974, Online 2306-0271, www.sne-journal.org

\begin{abstract}
Recently, there has been a growing interest in developing autonomous landing systems which is the greatest challenge of the whole autonomous flight mission. This paper presents market research results of currently available sensors for environment recognition during landing. Main focus is given to development of algorithms for the safe-landing zone estimation using LIDAR and stereo-camera sensors. And finally, the paper highlights challenges in this research field.
\end{abstract}

\section{Introduction}

A new revolutionary innovation in transportation is approaching which will allow traveling by air and will reduce both costs and time spent while increasing comfort. Similarly in the past, a bit more than a century ago first cars appeared that enabled the development of mass mobility. Nicolas-Joseph Cugnot built the first selfpropelled road vehicle, a military tractor running on steam, while the first gasoline powered car was invented by Karl Friedrich Benz in 1885. Recently five successful companies started partnership with Uber to elevate an electric flying taxi to service in 2020 [3]. One of them is also Slovenian company PIPISTREL VERTICAL SOLUTIONS d.o.o. [4], who also joined the student project PKP (entitled "By creative way towards knowledge"), and whose activity is summarized in this article.
The project is co-financed by the European Union from the European Social Fund and the Republic of Slovenia. Seven students and five mentors from the Faculties of electrical engineering and Economics in Ljubljana and the company PIPISTREL VERTICAL SOLUTIONS d.o.o. have cooperated in this project. The main purpose of this project is that students acquire additional competencies and knowledge that can help them with career and employment. During the project the students need to solve specific problems, and actual challenges defined by the participating company.

One of the key challenges of such autonomous VTOL (vertical takeoff and landing) aircrafts is the development of an automated and safe landing system. Before landing, the system needs to identify an appropriate landing surface where the aircraft can safely land. The identification of a safe landing zone cannot depend only on the pilot's view from the cabin as he may have incomplete information. Therefore we propose an automated sensor system that would assist the pilot, and in the future it will also allow autonomous operation. The proposed system is based on 3D LIDAR and stereo camera measurements, which are processed in the algorithms to obtain depth images of the environment. These sensors turned out to be currently the most appropriate selection for the task.

We decided to use and upgrade existing solutions due to the economies of scale, where the price of the final product decreases with demand on the market. That would also allow to offer the product to global market in the future. 
In the following, the overview of the existing sensors currently available on the market is given, followed by the description of the developed algorithms for sensor data processing, identification of landing zone from the sensor measurement and appropriate presentation of the results to the user. Realistic measurements were taken to test and validate the proposed ideas. Finally, some future challenges in the area of research are exposed.

\section{Landing Problem and Used Development Environments}

The main goal of the project was to propose a conceptual idea how to solve autonomous landing of VTOL aircraft. The proposal includes the selection of appropriate sensors and analysis of their possible placements to the aircraft, the required sensor measurements processing and development of the basic algorithms that would illustrate the main idea. First, the main assumption, starting points and limitations were defined as follows:

- $\quad$ the system needs to be operational for heights up to 50 meters,

- final decision for the landing must be given at least at a height of $15 \mathrm{~m}$,

- $\quad$ used sensors should not include mechanically spinning parts to increase robustness, sensors need to be fixed or only rotation in one direction is permitted,

- more sensors can be arranged over the hull of the aircraft,

- the whole cost of the sensor system should not exceed $10.000 €$

During the development, the following tools were used:

- MATLAB, where the main development of the algorithms was performed. Due to many libraries, easy work with matrices, easy debugging and visualization, it allows rapid development of algorithm ideas and processing of data. This environment was used to implement algorithms and for data processing from LIDAR and imaging camera.

- PCL (Point cloud library), which is an open source library for 2D and 3D image and data clouds processing. It contains algorithms for filtering, surface reconstruction (modelling and segmentation), object of known shape recognition etc. The library contains several sub libraries. It supports various sensors, such as stereo cameras, 3D scanners, Kinect and similar. PLC library was used in the project for processing images from stereo camera.

- Fusion 360, which is a 3D modelling or drawing program. Its advantage is that it contains all phases of product development (CAD, CAM in CAE) in a single platform. It is a free access program for students. We used it for drawing 3D models and also for the analysis of various scenarios of sensor selection and their positioning on the aircraft model.

\section{Sensor Overview for Autonomous Landing Purpose}

Sensors are the most important elements in autonomous landing systems. When selecting appropriate sensors, the preferred are those who have at least $30 \mathrm{~m}$ range, have no rotating mechanical parts and are sufficiently robust.

The main goal of the performed analysis is an overview of the available sensors in the market, identifying which of those are still in the development phase and will be available soon and their types. We have checked the entire offer (2D, 3D and 1D LIDAR, cameras, stereo cameras, IR cameras, radars, ToF cameras, ultrasound sensors, etc.) and selected the most appropriate ones for our needs (see Table 1).

We have found out that many suppliers offer solutions, which are still under development and as such unavailable and usually with uncomplete specifications. An example of such disappointment were Solid-State LIDAR sensors, which would be the optimal choice as they have no moving parts (e.g. rotating mirrors) and are therefore more robust. However, the majority of them is currently unavailable (still in development) or have a small field of view, short range, rough resolution and a high price.

On the contrary, a very high potential have imaging sensors (camera, stereo camera), which are already available and their measurement (image) includes a high amount of information. However, a bigger challenge when using cameras is the quality of image processing, in order to extract the desired information from the measurements, such as reliable 3D images or point clouds of the environment from larger distances. 
IR cameras operation depends on temperature contrast of the environment which can in a given area fluctuate a lot. Nevertheless, the camera returns a 2D image, suitable for processing and rapid analysis of potential objects. ToF cameras only operate at very short distances and in good conditions (clear weather). Their good side is that they can directly measure 3D information. Radars can operate on both short and long distances, but are limited by poor resolution.

The choice of currently the most suitable sensors for our solution, which are available on the market, is shown in Table 1.

Four different scenarios for landing area detection were suggested. They all share the same basic idea in acquiring a $3 \mathrm{D}$ cloud of space points, which is then processed to identify good and bad areas for landing.

\begin{tabular}{ll}
\hline Sensor type & Sensor \\
\hline Solid-State LIDER & Cepton HR80W \\
\hline Stereo camera & MultiSense S2 \\
\hline Infrared camera & NightHawk 2 \\
\hline Time of Flight & 03D303 \\
\hline Radar & Continental ARS4-A \\
\hline
\end{tabular}

Table 1: Selected sensors.

In the classification of the planes, the RANSAC (RANdom SAmple Consenses) method turned out as the most appropriate. Each scenario contains a combination of two different sensor principles where the weaknesses of the one is eliminated by the advantages of the other. The following combinations of sensors are made:

- solid-state LIDAR combined with stereo camera,

- IR camera combined with stereo camera,

- Radar combined with stereo camera and

- ToF camera combined with stereo camera.

All mentioned approaches are based on gradual measurements, where the use of the sensor at different heights improves the accuracy and reliability of the taken measurements. Integration of several sensors in a solution also improves redundancy, thereby increasing safety in the event of a malfunction. More information sources are obtained for fusion and comparison to other sources of information.
Some sensors, e.g. solid state LIDAR, have a very narrow range of measurement $(\mathrm{FoV})$ such as, for example, $60^{\circ} \times 24^{\circ}$ in the Cepton HR80W (Wide Angle) model. A possible solution (less robust) here is a mechanical mechanism that would move the sensor in a circular arc and thus capture several individual images, which are merged into a common image of the terrain. Another solution (more expensive and more demanding) is the installation of several identical sensors, which at the same time cover a larger area.

\section{Safe Landing Zone Estimation}

\subsection{Basic approach}

The block diagram of the data processing from the capture of the measurements to the graphic representation is given in Figure 2. It is constructed in a way that it is common to both the LIDAR measurements and the stereo camera measurement obtained by the constructed depth image.

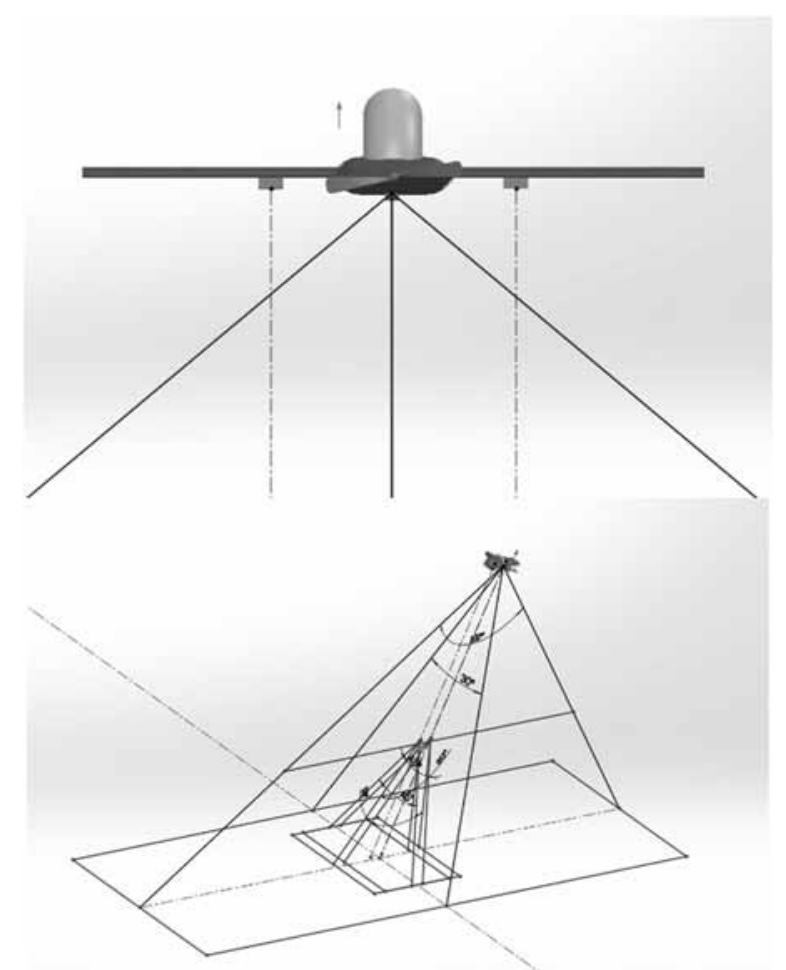

Figure 1: Sensor placement on the aircraft (LIDAR sensor on the middle and stereo cameras on the wings) and image from the analysis of obtained field of views. 


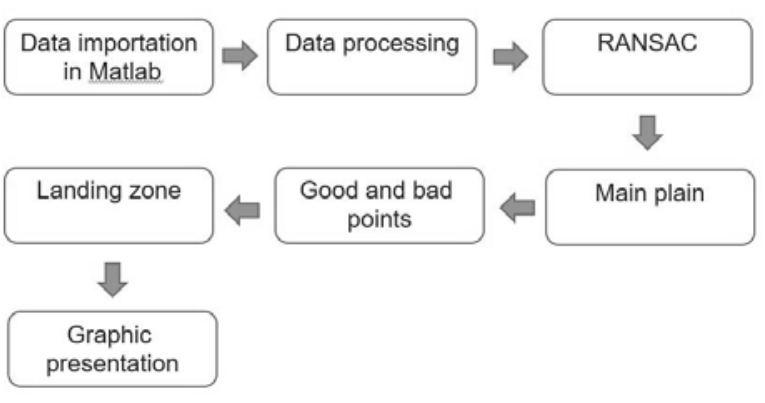

Figure 2: Work flow of data processing from captured measurements towards graphic presentation.

\subsection{Performing measurements}

Development and operation of algorithms were carried out exclusively on real measurements, which were captured from two different sensors, i.e. LIDAR (Velodyne VLP-16) and stereo camera (two ACME VR06 at a distance of $1 \mathrm{~m}$ ). Measurements were taken from a window on the second floor of the Faculty of Electrical Engineering (view of the parking lot, Figure 3).

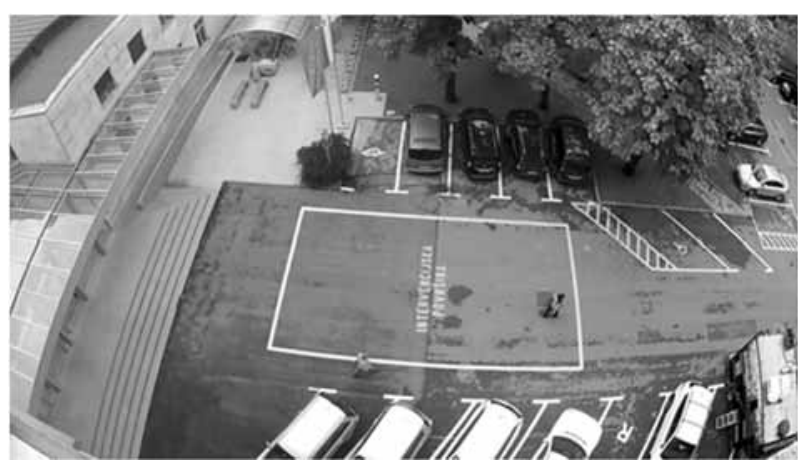

Figure 3: View of the area that was measured.

\subsection{Approach using LIDAR sensor}

Data from Velodyne VLP-16 LIDAR was obtained in the form of a pcap (Velodyne packet capture) file, which can be transformed into a csv (comma-separated values) format using VeloView.

We imported the data into MATLAB using the csvread function, where the result is a cloud of points given in the Cartesian coordinate system of the LIDAR sensor (Figure 4). The Velodyne VLP-16 measures $360^{\circ}$ field of view, but only a specific area of this measurement is interesting for our problem therefore irrelevant points were removed.

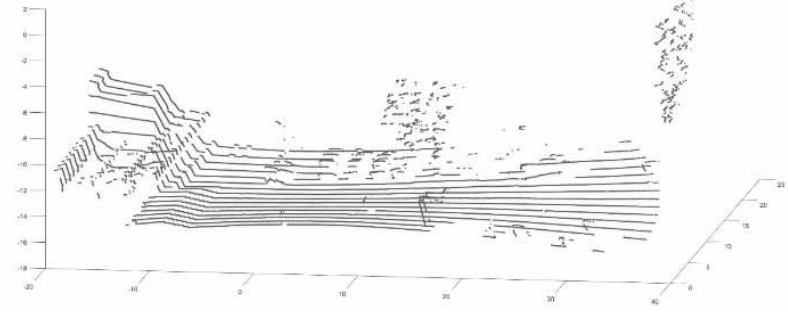

Figure 4: 3D depth image obtained from the LIDAR.

The brief idea of the implemented safe landing area algorithm is as follows. From the obtained measurement determine the boundaries of the area and divide it into sub-areas. Using the RANSAC method in each subregion, find a plain that fits best to the points in that region. RANSAC is an iterative process for estimation of the parameters of a mathematical model from data. Data can contain outliers which must not influence the estimation of parameters. The algorithm first checks if there are enough points, otherwise the sub-area is marked as an undefined area. If there are enough points, three points are randomly selected, which accurately determine a plane. In the next step, the distances of all points of the sub-area to the plane are calculated. All the points that are in a certain proximity (e.g. at least 10 $\mathrm{cm}$ ) of the plane are recorded and considered as good points. The described procedure is repeated n-times. We only save the list of points when their number is higher than in all previous experiments.

Good points can be classified also if they have been marked more than m-times as close enough to a randomly found plane.

After completion of the iterations, the largest list of good points is stored and the plane, which best fits the good points, is determined by the least-square method. The resulting plane is evaluated based on how close it is to the horizontal plane. It can also be validated by the alignment of the plane's normal vector with z-axis (of the scene) and by the total distance of all the good points from it. On the basis of deviations (from the normal direction and points distances), the obtained plane is estimated to be acceptable or unacceptable, or as a good or a bad zone.

In the next step, the main plane (in Figure 5-6, indicated by dark green) is determined taking into account all good planes of the sub-areas. This can be done in several different ways. Good plains at different heights must not be joined. 
Follows the classification of all points based on the distance from the main plane (with threshold e.g. 10 $\mathrm{cm}$ ) to determine if points are good or bad. Additionally the surroundings of all bad points are checked where the first good point is searched around a given bad, which is subsequently interpreted as a bad point. This creates information on the edges of the obstacles. All the given points are mapped into the main plane, where new "undefined" points are added in the areas where the points are too far away from each other. New undefined points do not contain any other points in the radius $\mathrm{R}$.

Then the largest radius containing good points is searched to obtain a candidate for the best landing surface. Additionally, the whole searched area needs to be within the boundaries of the main plane and must not be in contact with an undefined area. The search of the best landing area is an iterative process where the search area moves along $x$ and $y$ axes and saves the most successful area found.

The final result of the mentioned recognition process is the graphic presentation (Figure 5, first method) of the main plane with the illuminated circle (light green) representing the suitable landing zone, and a slightly darker green color on the plane shows good points and the red color shows the obstacles.

In the second presentation approach (Figure 6) the results are calculated first in each sub-region, by calculating the average value by the median. In the undetermined regions, this average value is determined from the median of the neighborhood average values. Then all these obtained points are connect to form a continuous surface.

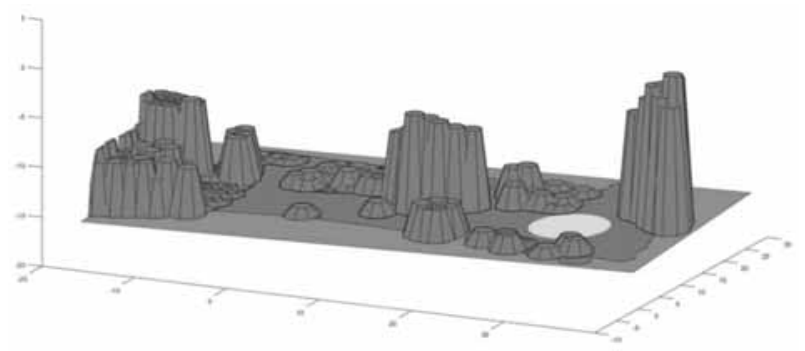

Figure 5: Presentation of the results obtained by the first method.

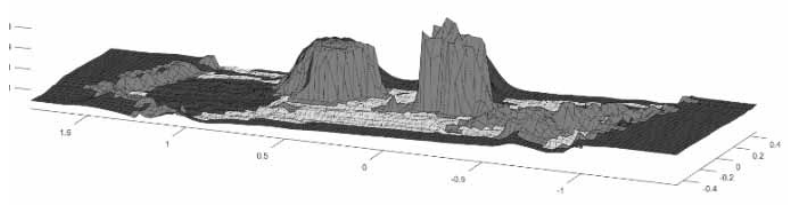

Figure 6: Presentation of the results obtained by the first method.

\subsection{Stereo camera approach}

Using the stereo camera which consists of two ACME VR06 cameras (placed $1 \mathrm{~m}$ apart), measurements of the same environment (Figure 3) as in the case of LIDAR were performed. After applying appropriate calibration of the stereo camera, a corresponding depth image is obtained. It turned out that a quality of calibration is of great importance in obtaining a proper depth image. In the following, the process of obtaining depth images is described.

The first step in image processing is the calibration of the stereo camera. The calibration provides parameters describing the camera's internal parameters and external parameters. The internal parameters represent the optical center and focal length of the camera. While the external parameters represent the orientation and distance transformation between the two cameras. Due to the use of cameras with a wide angle, it is important to use radial coefficients of distortion, which take into account the grater deviations of the light rays at the edges of the lens as compared to the optical center. As usually, the calibration was performed using a chessboard image that is in the field of view of both cameras. In each image, the vertices of the chessboard are searched. By using several image frames with marked vertices and information on the true distance between individual vertices, the necessary internal and external parameters as well as the distortion parameters can be calculated following the algorithm described in article [1].

By using calibration parameters, the distortion in the images can be corrected and compensated. Further on by matching the images, it is achieved that the corresponding points in both images are in the same row, or the same values of the y coordinate of the image.

In order to achieve this, a transformation of the image is done, which makes the same result as it would be obtained if the cameras were parallel and at the same height. By obtaining transformed images, a picture of the disparity between the two images is calculated. The disparity represents the difference in the pixels between the columns of the correspondence points on the left and the right image.

Calculation of the disparity was carried out using semi-global matching method [2]. This method differs from the usual methods for disparity calculation because it requires a similar disparity between the nearby blocks (groups) of pixel elements. 
This can partially eliminate noise, which appears due to the lack of image elements in the search for a disparity.

The cloud of points from the found pairs of image pixels is obtained by the following transformation:

$$
\left[\begin{array}{cccc}
1 & 0 & 0 & -c_{x} \\
0 & 1 & 0 & -c_{y} \\
0 & 0 & 0 & f \\
0 & 0 & -1 / T_{x} & \left(c_{x}-c_{x}^{\prime}\right) T
\end{array}\right]\left[\begin{array}{l}
x \\
y \\
d \\
1
\end{array}\right]=\left[\begin{array}{c}
X \\
Y \\
Z \\
W
\end{array}\right]
$$

where $c_{x}$ and $c_{x y}$ are the center of the left camera sensor, $f$ is the focal length of the left camera, $T$ is the distance between the cameras, $c_{x}^{\prime}$ is the $x$-coordinate of the center of the right camera and $d$ is the dispersity. Since we deal with homogeneous coordinates, the pixel position in $3 \mathrm{D}$ coordinates is equal to $(X / W, Y / W, Z / W)$.

When the point cloud is obtained, it is processed in the same manner as with the LIDAR data (Figure 2 and Subsection 3.3). The result of the processing is shown in Figure 7.

Each point of the depth image in Figure 7 represents $10 \times 10$ pixels in the original image. If the point is good it is colored green or if it is bad it is colored red. In the case where the point in Figure 7 is not specified, the corresponding image elements are shown in black.

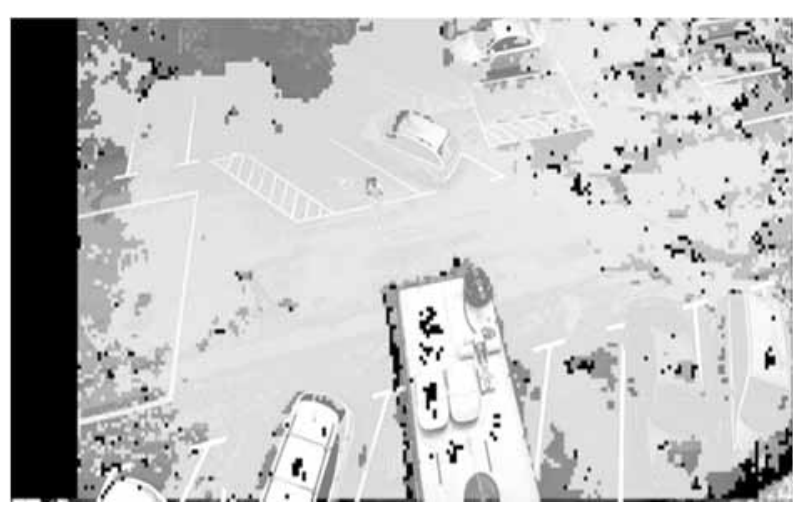

Figure 7: Depth image obtained from the stereo camera. View of good (green) zones, bad (red) zones and undefined (black) zones on the left camera image.

\section{Conclusion}

The paper presents a proposal for the design of an autonomous landing system and an automatic estimation of the safe zone based on 3D LIDAR and stereo cameras. Based on the performed overview of sensors it is concluded that many sensors that are suitable (e.g. solidstate LIDAR) are still in the development phase.
This study highlights mainly the limitations and advantages of the available sensors currently on the market.

The results obtained in the evaluation of the proposed algorithms operation are good, considering the expected problems in machine vision even above expectations. Algorithms obtained in this phase of research cannot run in real time, as the primary goal was to validate proposed concepts, algorithms, and a set of sensors. The obtained results of the research suggest that it is possible to obtain a reliable autonomous zone detection system using available and affordable sensors and existing algorithms.

Further improvements and steps for future development would be the following. Validate the proposed methodology also on higher quality sensors, especially cameras. Upgrade of the algorithms to obtain greater robustness, by choosing more reliable features in determining image disparities and optimization to achieve real-time operation. Acquisition of real measurements with the proposed sensors on real aircraft and performing possible improvements in the sensors positioning, their selection and in recognition algorithms.

\section{Acknowledgement}

The presented work was performed in the frame of PKP project, which is co-financed by the European Union from the European Social Fund and the Republic of Slovenia.

\section{References}

[1] Zhengyou T. A flexible new technique for camera calibration. IEEE Transactions on pattern analysis and machine intelligence. 2000; vol. 22: pp.1330-1334.

[2] Hirschmuller H. Accurate and Efficient Stereo Processing by Semi-Global Matching and Mutual Information. International Conference on Computer Vision and Pattern Recognition. 2005; vol. 2: pp. 807-814.

[3] Uber Elevate, Fast-Forwarding to a Future of OnDemand Urban Air Transportation, available at: https://www.uber.com/elevate.pdf, 2016.

[4] Pipistrel d.o.o., Uber partners with Pipistrel Aircraft to manufacture electric VTOLs, available at: https://www.pipistrel.si/news/uber-partners-withpipistrel-aircraft-to-manufacture-electri, 2017 